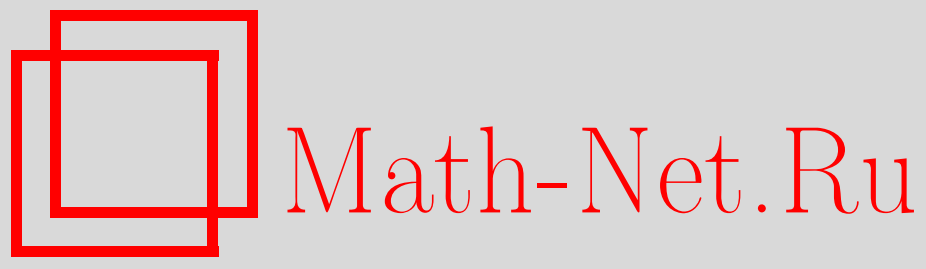

С. Б. Табалдыев, Аддитивность гомологических размерностей для некоторого класса банаховых алгебр, Функи. анализ и его прил., 2006, том 40, выпуск $3,93-95$

DOI: https://doi.org/10.4213/faa751

Использование Общероссийского математического портала Math-Net.Ru подразумевает, что вы прочитали и согласны с пользовательским соглашением

http://www . mathnet.ru/rus/agreement

Параметры загрузки:

IP : 52.6 .47 .48

26 апреля 2023 г., 12:56:05

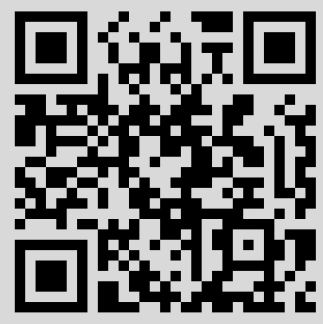


http://arxiv.org/math.RT/0012220. [5] И. М. Гельфанд, Г. Е. Шилов, Обобщенные функции. Bып. 2. Пространства основных и обобщенных фбункций, Физматгиз, М., 1958.

Московский центр непрерывного

Поступило в редакцию математического образования

e-mail: stoyan@mccme.ru

8 февраля 2005 г.

УДК 517.98

\title{
Аддитивность гомологических размерностей для некоторого класса банаховых алгебр*
}

\author{
(c) 2006. С. Б. ТАБАлдЫЕВ
}

В 1997 г. Ю. В. Селиванов в [1] доказал следующие «формулы аддитивности» для гомологических размерностей (определения см. ниже): если $A-y н u-$ тализация бипроективной коммутативной банаховой алгебры с бесконечным спектром, а $B$ - произвольная унитальная банахова алгебра, то $\operatorname{dg} A \widehat{\otimes} B=$ $\operatorname{dg} A+\operatorname{dg} B u \operatorname{db} A \widehat{\otimes} B=\operatorname{db} A+\operatorname{db} B$.

Пусть $\Omega$ - бесконечное метризуемое компактное топологическое пространство, у которого производное множество некоторого конечного порядка пусто. (Напомним [2], что производное множество топологического пространства есть множество всех его предельных точек.) Пусть $A=C(\Omega)$ - банахова алгебра всех непрерывных функций на $\Omega$ с равномерной нормой. Мы утверждаем (в теореме 2), что формулы аддитивности верны и в этом (новом) случае; легко убедиться в том, что если $C(\Omega)$ - унитализация бипроективной алгебры, то уже его производное множество первого порядка $\Omega^{\prime}$ состоит из одного элемента, а производное множество второго порядка $\Omega^{\prime \prime}=\left(\Omega^{\prime}\right)^{\prime}$ пусто.

Пусть $X$ - левый банахов $A$-модуль, а $E$ - банахово пространство. Тогда тензорное произведение $X \widehat{\otimes} E$ является левым банаховым $A$-модулем относительно внешнего умножения, определяемого формулой $a \cdot(x \otimes u)=(a \cdot x) \otimes u$ для $a \in A, x \in X$ и $u \in E$. (Определение проективного тензорного произведения $\widehat{\otimes}$ банаховых пространств и проективного тензорного произведения $\widehat{\otimes}_{A}$ левого и правого банаховых модулей над банаховой алгеброй $A$ можно найти, например, в книге [3].)

Предположим, что $A$ - унитальная банахова алгебра, а $X$ - унитальный левый банахов $A$-модуль. Напомним, что модуль $X$ называется проективным (см. [3]), если он является прямым слагаемым $A$-модуля $A \widehat{\otimes} E$ для некоторого банахова пространства $E$. Аналогично определяется проективный унитальный банахов $A$-бимодуль. Напомним также, что длина самой короткой проективной допустимой (т. е. расщепимой как комплекс банаховых пространств) резольвенты $A$-модуля $X$ называется его гомологической размерностью и обозначается через $A$ dh $X$. Гомологическая размерность банахова $A$-бимодуля определяется аналогично. Верхняя грань гомологических размерностей унитальных левых

*Работа выполнена при финансовой поддержке Российского фонда фундаментальных исследований (грант 05-01-00982). 
банаховых $A$-модулей называется глобальной размерностью алгебры $A$. Гомологическая размерность банахова $A$-бимодуля $A$ называется биразмерностью алгебры $A$. Глобальная размерность алгебры $A$ обозначается через $\operatorname{dg} A$, а биразмерность - через $\mathrm{db} A$.

Нетрудно доказать, что в пространстве $\Omega$ всюду плотно множество всех его изолированных точек. Выберем произвольные точку $\omega \in \Omega^{\prime}$ и последовательность попарно различных точек $s_{n} \in \Omega \backslash \Omega^{\prime}$, сходящуюся к $\omega$. Пусть $A_{\omega}=\{f \in C(\Omega) \mid f(\omega)=0\}$. Банахово пространство $\ell^{\infty}$ ограниченных последовательностей является левым банаховым $C(\Omega)$-модулем относительно внешнего умножения $a \cdot x=\left\{a\left(s_{n}\right) x_{n}\right\}$, где $a \in C(\Omega), x=\left\{x_{n}\right\} \in \ell^{\infty}$.

Теорема 1. Если $B$ - произвольная унитальная банахова алгебра, а $X-$ произвольный унитальный левый банахов $B$-модуль, то

$$
C(\Omega) \widehat{\otimes} B \operatorname{dh} \ell^{\infty} \widehat{\otimes} X=2+{ }_{B} \operatorname{dh} X\left(={ }_{C(\Omega)} \operatorname{dh} \ell^{\infty}+{ }_{B} \operatorname{dh} X\right) .
$$

ДоказАтельство. Рассмотрим левый $C(\Omega)$-модуль $c_{e}=A_{\omega} \widehat{\otimes}_{C(\Omega)} \ell^{\infty}$ и морфизм левых банаховых $C(\Omega)$-модулей $\kappa: c_{e} \rightarrow \ell^{\infty}$, определяемый формулой $\kappa\left(a \otimes_{C(\Omega)} x\right)=a \cdot x$ для $a \in A_{\omega}$ и $x \in \ell^{\infty}$.

Согласно [4, лемма 9.1], левый банахов $C(\Omega)$-модуль $\ell^{\infty}$ имеет такую допустимую резольвенту $0 \longleftarrow \ell^{\infty} \longleftarrow Q \longleftarrow P \longleftarrow A_{\omega} \widehat{\otimes} c_{e} \longleftarrow 0$ длины 2 , что

$$
\begin{gathered}
Q=\left(C(\Omega) \widehat{\otimes} \ell^{\infty}\right) \oplus c_{e}, \quad P=\left(C(\Omega) \widehat{\otimes} c_{e}\right) \oplus\left(A_{\omega} \widehat{\otimes} \ell^{\infty}\right), \\
\partial(a \otimes y)=(a \otimes y, a \otimes \kappa(y)) \quad\left(a \in A_{\omega}, y \in c_{e}\right) .
\end{gathered}
$$

Очевидно [3, предложение II.3.13], что $C(\Omega)$-модуль $c_{e}$ топологически изоморфен подмодулю $c_{0} \subset \ell^{\infty}$, состоящему из всех последовательностей, стремящихся к нулю. Так как последовательность $s_{n}$ сходится к $\omega$ и состоит из изолированных точек, то нетрудно заметить, что модуль $c_{0}$ изоморфен идеалу всех функций из $C(\Omega)$, которые равны 0 при любом $s \in \Omega, s \neq s_{n}(n=0,1, \ldots)$. Поскольку $\Omega$ метризуемо, банаховы $C(\Omega)$-модули $A_{\omega}$ и $c_{e}$ являются проективными [3, теорема IV .2.13]. Следовательно, указанная выше резольвента проективна.

Если ${ }_{B}$ dh $X$ есть 0 либо $\infty$, то результат вытекает из [1, следствие 3.14]. Если ${ }_{B} \mathrm{dh} X=n$, где $0<n<\infty$, выберем проективную резольвенту

$$
0 \longleftarrow X \longleftarrow P_{0} \longleftarrow \cdots \longleftarrow P_{n-1} \longleftarrow P_{n} \longleftarrow 0
$$

модуля $X$. Согласно [1, следствие 3.2], мы имеем $C(\Omega) \widehat{\otimes} B$ dh $\ell^{\infty} \widehat{\otimes} X \leqslant 2+n$. Предположим, что $C(\Omega) \widehat{\otimes} B$ dh $\ell^{\infty} \widehat{\otimes} X<2+n$. Тогда, используя [1, предложение $3.18]$, получаем, что морфизм левых $C(\Omega) \widehat{\otimes} B$-модулей

$$
\Delta: A_{\omega} \widehat{\otimes} c_{e} \widehat{\otimes} P_{n} \longrightarrow\left(C(\Omega) \widehat{\otimes} c_{e} \widehat{\otimes} P_{n}\right) \oplus\left(A_{\omega} \widehat{\otimes} \ell^{\infty} \widehat{\otimes} P_{n}\right) \oplus\left(A_{\omega} \widehat{\otimes} c_{e} \widehat{\otimes} P_{n-1}\right),
$$

определяемый формулой

$\Delta(a \otimes y \otimes z)=(a \otimes y \otimes z, a \otimes \kappa(y) \otimes z, a \otimes y \otimes \psi(z)) \quad\left(a \in A_{\omega}, y \in c_{e}, z \in P_{n}\right)$, имеет левый обратный. Теперь из [1, Proposition 4.6] мы получаем, что морфизм $\psi: P_{n} \rightarrow P_{n-1}$ имеет левый обратный морфизм левых $B$-модулей. Отсюда ${ }_{B} \mathrm{dh} X<n$, а это противоречит предположению.

В работе [5] было доказано, что если $\Omega$ - бесконечный метризуемый компакт, у которого производное множество некоторого конечного порядка пусто, 
то $\operatorname{dg} C(\Omega)=\operatorname{db} C(\Omega)=2$. С помощью этого результата, теоремы 1 и некоторых оценок из работы [1] доказывается следующая

Теорема 2. Если $B$ - произвольная унитальная банахова алгебра, то

$$
\begin{aligned}
& \operatorname{dg} C(\Omega) \widehat{\otimes} B=\operatorname{dg} C(\Omega)+\operatorname{dg} B(=2+\operatorname{dg} B), \\
& \operatorname{db} C(\Omega) \widehat{\otimes} B=\operatorname{db} C(\Omega)+\operatorname{db} B(=2+\operatorname{db} B) .
\end{aligned}
$$

Теорема 2 и следствие 3.16 из [1] сразу доставляют

СлЕДСТВИЕ 1. Пусть $\Omega_{i}(i=1,2, \ldots, n)$ - метризуемые компакты, у каждого из которых производное множество некоторого конечного порядка пусто. Пусть $A=C\left(\Omega_{1}\right) \widehat{\otimes} \cdots \widehat{\otimes} C\left(\Omega_{n}\right)$ - проективное тензорное произведение банаховых алгебр $C\left(\Omega_{i}\right)$. Тогда $\operatorname{dg} A=\operatorname{db} A=2 m$, где $m-$ число бесконечных пространств среди $\Omega_{i}$.

СЛЕДСТвИЕ 2. Пусть $A$ - такая же банахова алгебра, как в следствии 1, a $B$ - произвольная унитальная банахова алгебра. Тогда $\operatorname{dg} A \widehat{\otimes} B=\operatorname{dg} A+\operatorname{dg} B$ $u \mathrm{db} A \widehat{\otimes} B=\mathrm{db} A+\mathrm{db} B$.

Автор благодарен Ю. В. Селиванову и А. Я. Хелемскому за постановку задач и полезные обсуждения.

\section{ЛитерАТУРА}

[1] Yu. V. Selivanov, in: Banach Algebras'97 (eds. E. Albrecht and M. Mathieu), Walter de Gruyter, Berlin, 1998, 441-459. [2] К. Куратовский, Топология, т. 1, Мир, М., 1966. [3] А. Я. Хелемский, Гомология в банаховых и топологических алгебрах, Изд-во МГУ, М., 1986. [4] А. Я. Хелемский, Труды семинара им. И. Г. Петровского, 3 (1978), 223-242. [5] S. B. Tabaldyev, Bull. London Math. Soc., 37 (2005), 927-932.

Московский государственный технический университет им. Баумана

Поступило в редакцию e-mail: seytek@newmail.ru 